Kalpa Publications in Engineering
Volume 3, 2020, Pages 109-117
Proceedings of International Sym-
posium on Applied Science 2019

\title{
Research on the production of optical needles used in intra-venous laser
}

\author{
Ngoc An Dang Nguyen ${ }^{1}$, Anh Tu Tran ${ }^{1}$, To Ni Phan Van ${ }^{1}$, Trung Nghia \\ $\operatorname{Tran}^{1}$ and Minh Thai Tran ${ }^{1}$ \\ ${ }^{1}$ Ho Chi Minh City University of Technology - VNUHCM \\ dnnan.sdh19@hcmut.edu.vn
}

\begin{abstract}
Nowadays, fiber optical is used in many areas such as information transmission, medical with the advantage is rapid and avoid the loss of transmission efficiency. In medical use, the laser is increasingly used in rehabilitation treatment, especially low-level laser with the biological responses. Optic needle is one of the optimum options for bringing low-level lasers into the body through the intravenous route to interact with blood cells in blood vessels. We propose the application of fiber, the first step is the single-mode fiber in the low-level semiconductor red laser beam with wavelength 632,8 $\sim 680 \mathrm{~nm}$ into the vascular veins to provide effective low-level laser treatment. This paper reported the research on the production of intravenous optical needle.
\end{abstract}

Keywords: Intravenous, Low level Laser Therapy (LLLT), Near infrared ...

\section{Introduction}

In rehabilitation therapy, low-level lasers are widely used in treatment through the biological stimulation effect. In medical literature of the world has demonstrated seven stimulation responses such as anti-inflammatory, anti-inflammatory, cellular response, regenerative response, immune response, cardiovascular response, and endocrine response. Intravenous laser is used extensively to increase immune response, the cardiovascular system through an optical conductor for $650 \mathrm{~nm}$ wavelength.

In Vietnam, the first semiconductor laser was a semiconductor laser developed by the Laser Technology Laboratory (Ho Chi Minh City University of Technology - VNU-HCM) since 1985 and successfully manufactured in 1988.

From 1988 up to now, the equipment has been constantly improved, and also made more types of low-level laser therapy devices using the semiconductor laser, intravenous laser, ... In this research, 
we simulated and conducted the fiber-optic testing for intravenous laser therapy, which we call intravascular laser needle.

\section{Background}

\subsection{Introduction of intravenous low-level laser device}

The intravenous low-level laser device was developed in the direction of treating some cardiovascular diseases. The intravenous low-level laser device development has done after the successful optical fiber fabrication. The output power varies from the minimum value to the maximum value it achieves. This allows the therapist to choose the right capacity for the treatment of each patient.

It is possible to perform beam frequency modulation. This allows you to select the appropriate frequency for each disease. The semiconductor laser is the smallest intravenous laser device.

\subsection{Mechanism of intravenous low-level laser therapy}

The intravenous low-level laser therapy effects directly on the circulatory components, including heart, blood vessels, blood.

Intravenous low-level laser treatment has been successful for heart disease such as myocardial infarction, ischemic heart disease; fast-paced play; left ventricular dysfunction in progressive chest pain; endocarditis; acute myocarditis; chest pain; ...

For blood vessels: the blood vessels are more relaxed because:

- the diameter of the vein will expand;

- atherosclerosis decreases considerably.

For blood quality:

- reduces platelet aggregation;

- activation of the fibrinolytic system;

- optimized blood lipid spectrum.

As blood circulation improves, resulting in a series of body effects such as:

- adjusted specific and non-specific immune system;

- regulate the endocrine system;

- enhanced antioxidant activity;

- increases the ability to combine oxygen with red blood cells, increasing oxygen transport capacity in the blood;

- reduces platelet aggregation, activates fibrinolysis;

- anti-vibration, anti-arrhythmia, blood pressure.

\subsection{Intravenous low-level laser device}

Figure 1 shows the Intravenous low-level laser equipment manufactured by Laser Technology Laboratory, Ho Chi Minh City University of Technology-VNUHCM.

The wavelength of the semiconductor laser wavelength is $650 \mathrm{~nm}$.

- The output power: $0-5 \mathrm{~mW}$

- The frequency modulation of the beam varies from 5 to $100 \mathrm{~Hz}$. The $50 \mathrm{~Hz}$ modulation frequency is chosen as the standard frequency for treatment.

- The treatment part consists of two parts: the treatment time settings are 5, 10,15, 20, 25, 30, 35 and 40 minutes and the treatment time count.

- The power supply for the device: DC $12 \mathrm{~V}$ 


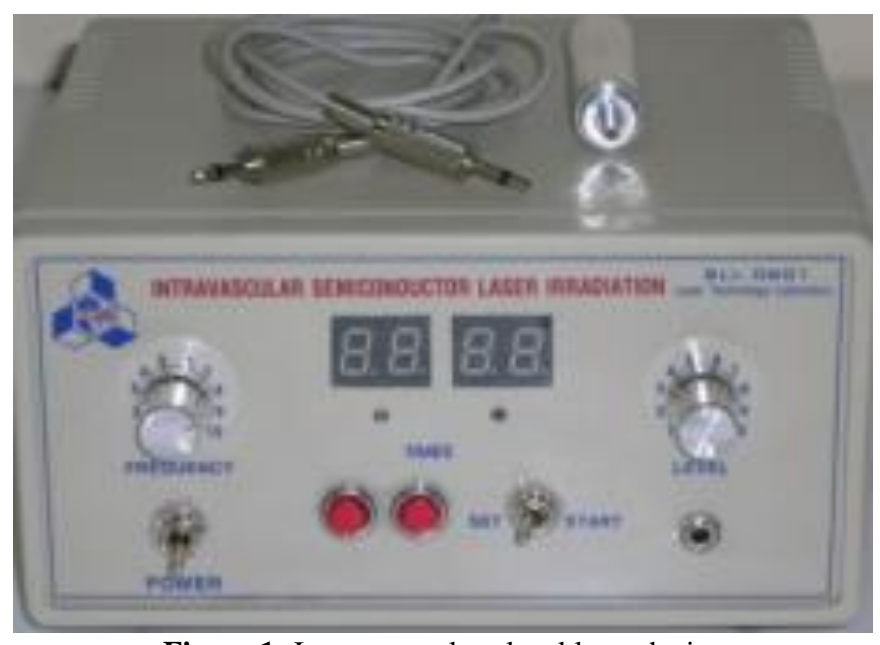

Figure 1: Intravenous low-level laser device

The treatment device consists of one controlled channel laser working at $650 \mathrm{~nm}$ wavelength, with the following main parameters:

- The output power varies from $(0-5 \mathrm{~mW})$.

The modulation frequency of the beam varies from $(5-100 \mathrm{~Hz})$.

- The above changes are intended for the following two treatment modalities:

a. treatment in the traditional Chinese style of acupuncture: treatment of chronic diseases using complementary techniques; treatment of acute illness using the technique;

b. Modulation based on modulation frequency: the modulation frequency $50 \mathrm{~Hz}$ should be used. On the device, there are two knobs to make the change on the power and the frequency.

Supply voltage: $12 \mathrm{~V}$ DC to improve the safety of the device.

\section{Optical needle used in intravenous laser therapy}

\subsection{Procedure for the use of the optical needle during treatment}

The supplies used for internal intravenous laser procedures include optical needle and $22 \mathrm{G}$ catheter. Use a $22 \mathrm{G}$ catheter to penetrate into the vein, then insert the needle into the vein through the catheter. The laser beam from the device transmits through the fiber and interacts with the blood cells.

The optical needle is connected to a low-level laser (650 nm wavelength) via a catheter $(\sim 4 \mathrm{~cm})$. First, the catheter is pierced through the patient's skin. The optical fiber blocks the catheter's entrance so that the blood can not leak and allow the laser to interact with the red blood cells.

Figure 2 shows the diagram of using the optical needle in the invascular low-level laser therapy. 


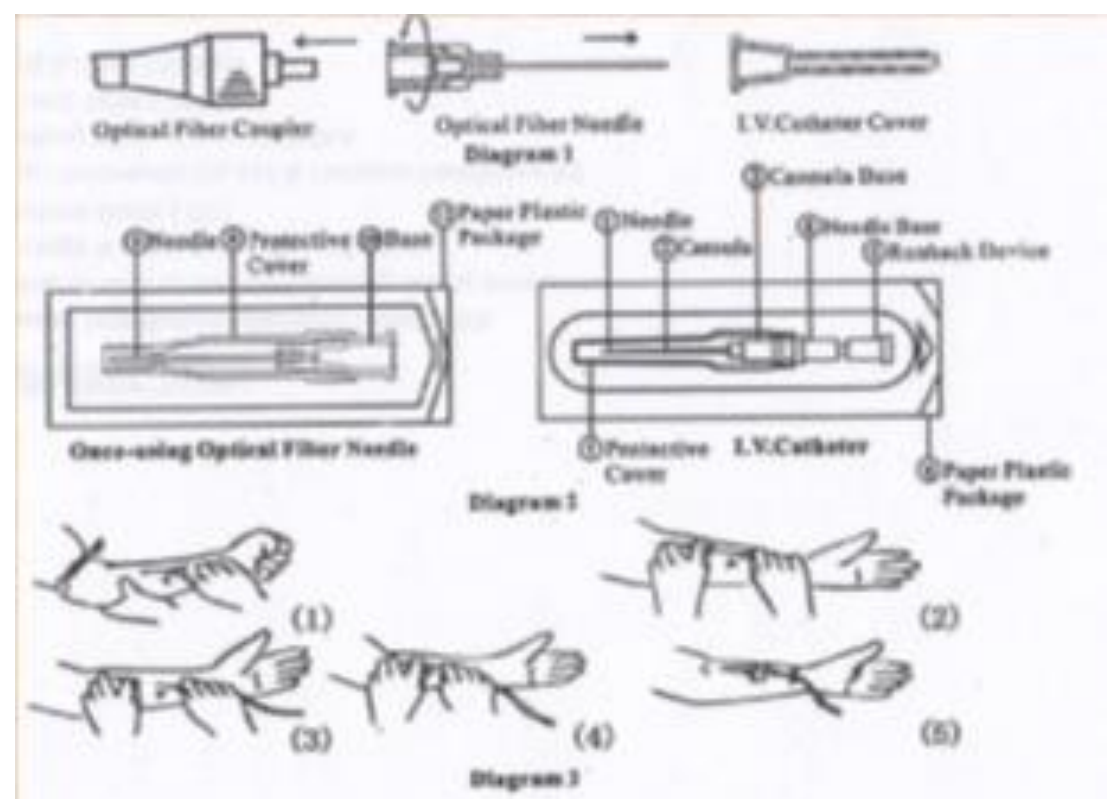

Figure 2: Diagram of using fiber optical needle in the invascular low-level laser therapy

Figure 3 shows the use of optical needle in clinical. Figure 4 shows the fiber optical needle kit.

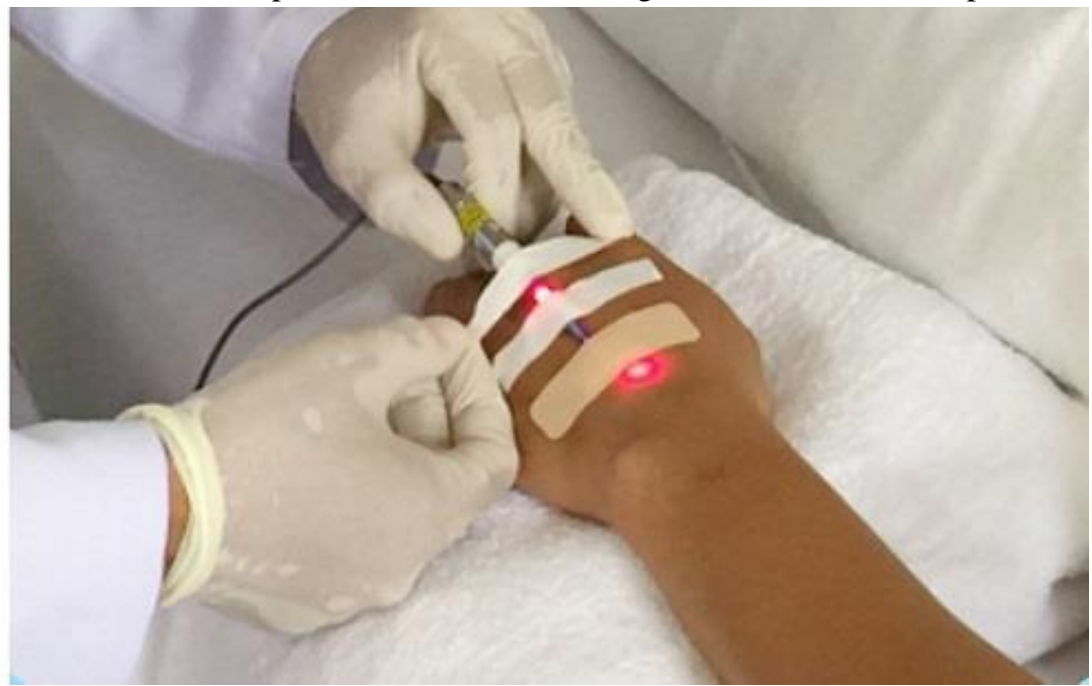

Figure 3: Paitents therapied by the intravenous low-level laser 


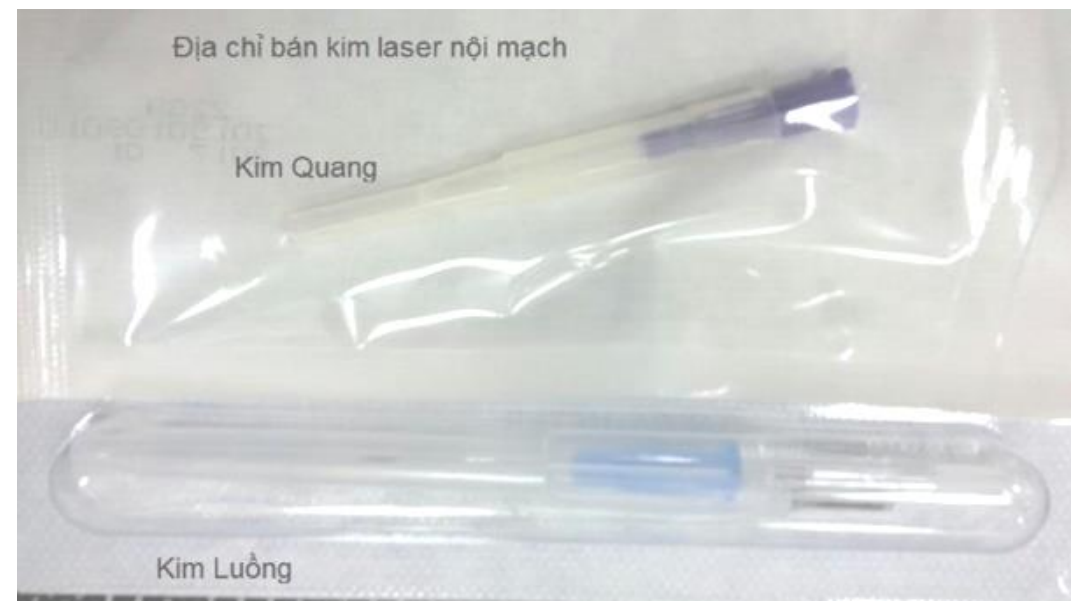

Figure 4: Fiber optical needle set used in the intravenous low-level laser therapy

\subsection{Research on the production of optical needle used in intravenous laser and trial production.}

Optical needle structure: optical fiber main component and connector hub to connect with the laser head. The optical fiber (single mode fiber) $0.5 \mathrm{~mm}$ diameter was used; the catheter using 22-Gauge IV catheter (I.D 0.6x25mm Terumo - Japan)

Processing plan for producing the optical needle used in internal intravenous laser as shown in the diagram below:

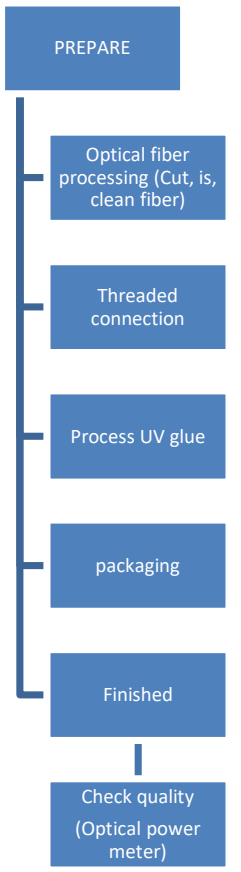

Diagram: Processing and testing needle production 


\section{Input preparation:}

1. Optical fiber;

2. Fiber optic cutter;

3. UV Adhesive - Loctite 3321TM;

4. Strap, catheter cover with PP plastic;

5. UV light to dry the glue.

\section{Prepare test equipment:}

1. 650nm wavelength laser light with two pulse and continuous modes;

2. Optical intensity meter.

\section{Prepare the finished product:}

1. EO bags for packing;

2. Super pocket press machine.

\section{Procedure:}

Step 1: Fabrication of fiber. Cut the fiber to a length of $3-4.5 \mathrm{~cm}$ with a dedicated knife for plastic fiber.

Step 2: Laser optical processing to complete the laser source. Clean fiber with antiseptic.

Step 3: Connect fiber optic and plastic back. Use UV glue to bond the fiber and sub strand.

Step 4: Sterilize the needle with sterilization, drying the product.

Step 5: Packaging products, sterilized EO (Ethylene Dioxide). Put to use.

\section{Fiber Optic Selection:}

Here we use single mode fiber, plastic cover, $0.5 \mathrm{~mm}$ diameter. Fiber optic head is machined with fiber optic head: One end of the optic head is attached to the optical receiver when it is placed in a plastic bag.

2. Select UV glue with the characteristics of the glue composition adapted to certain wavelengths. This type of UV glue is compatible with plastic.

- In the production research we use UV glue (LOCTITE TM) with a drying time of $\leq 5 \mathrm{~s}$ with UV light between Polycabonate with PVC, 30mW / cm2, 365nm [1]

- The UV lamp with peak at $365 \mathrm{~nm}$ wavelength, consistent with the characteristics of UV glue.

- Low-level semiconductor laser source was selected, $650 \mathrm{~nm}$ wavelength, semiconductor laser.

3. Laser power meter: Brand: Sanwa - Japan

- Model: mobiken - LP1

- Specifications: Measuring 650nm wavelength adaptive capacity, and (400nm -1100nm)

- Max 40mW- min: 40uW

Figure 5 shows the laser power meter. 

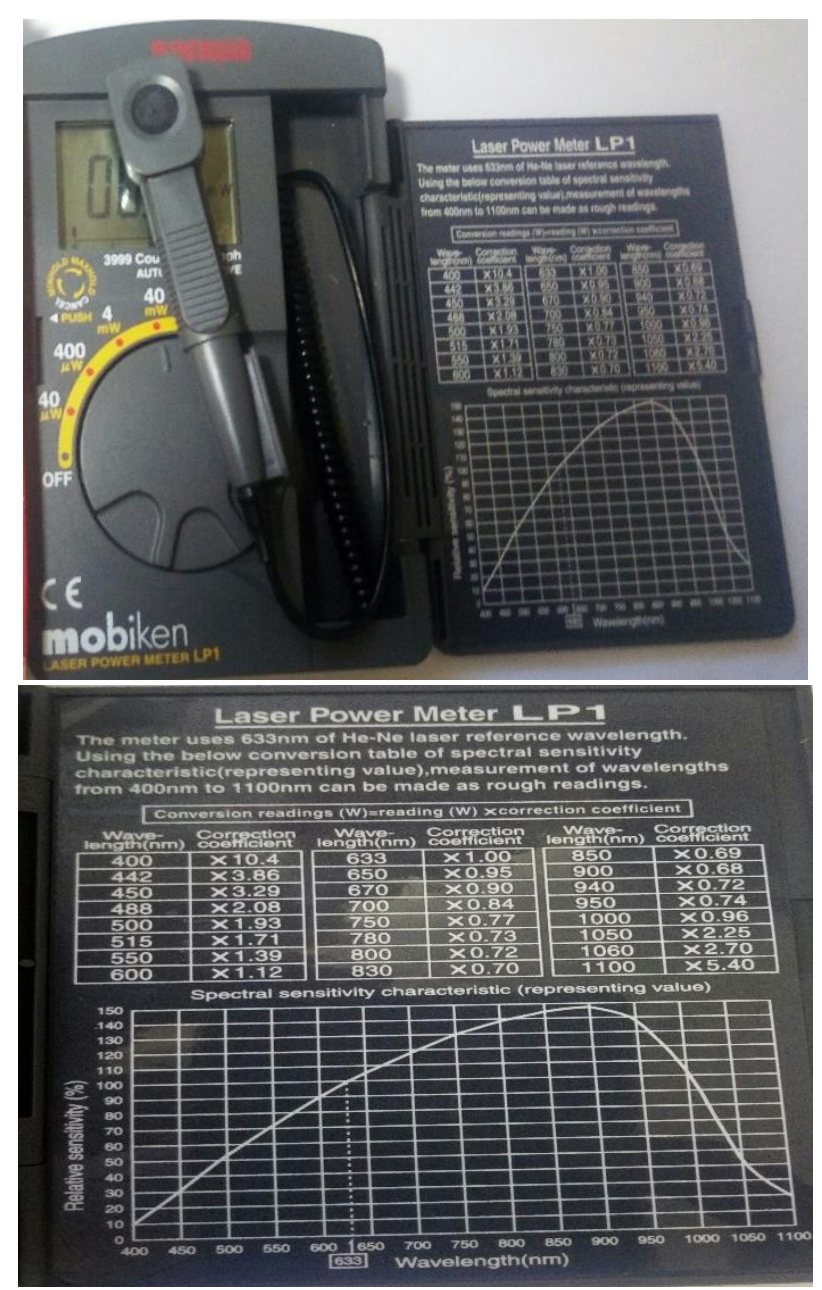

Figure 5: Laser power meter

4. Low temperature steam sterilization system E.O (Ethylene Oxide)

Sterilization of medical instruments that can not withstand high temperatures, sensitive to temperature and humidity. Because the needle is mainly plastic, it is only sterilized at low temperature. We choose to use EO gas with the reason:

- high sterilization efficiency;

- No contraindications with tube diameter and tool length.

- Low cost for 1 batch.

- Chemicals use $100 \%$ Ethylen oxide gas (EO) in a small gas tank, installed in the engine room, 1 bottle / batch, single dose. Shelf life of chemicals ( 5 years)

- Simple EO adsorption process and EO topping the table for penetration through complex medical materials. 


\subsection{Implementation Results}

After implementing the trial production process on the actual product. Through the test steps we have the initial results as follows:

1. Test optical test results through optical fiber

- Input: semiconductor laser source

- Output power 10mw, Wavelength: $650 \mathrm{~nm}$

- Optics: diameter $0.5 \mathrm{~mm}$, length: $4 \mathrm{~cm}$.

- Specifications: diameter $5 \mathrm{~mm}$, diameter $3 \mathrm{~mm}$, color: dark purple

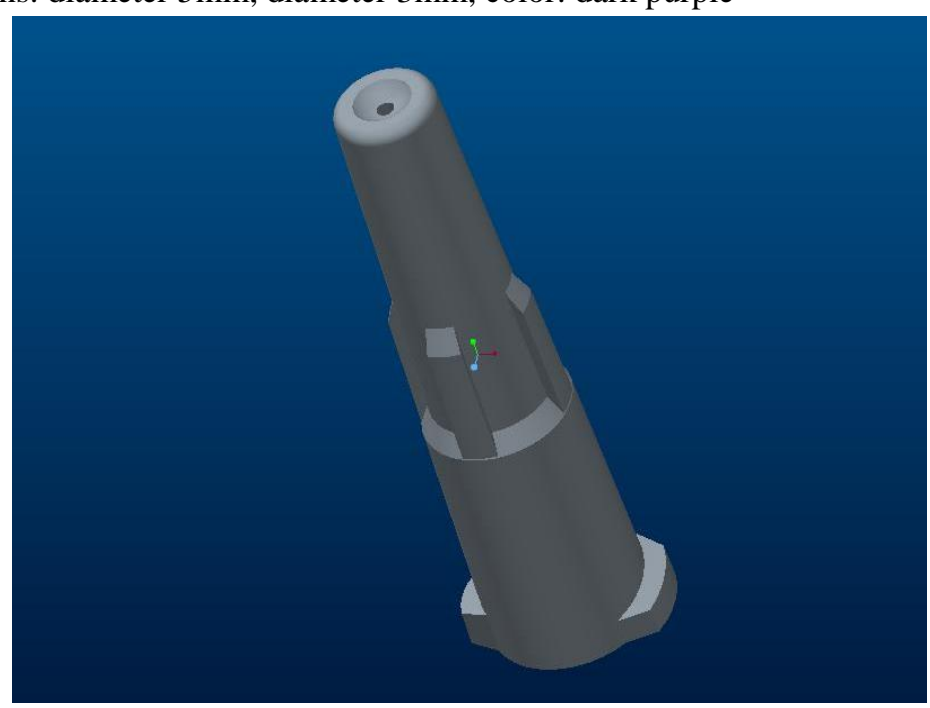

Figure 6: Simulated 3D connected to optical fiber

Testing results with a needle. Randomly take 30/1000 samples and test the remaining energy through optical fiber as follows:

\begin{tabular}{|c|c|c|}
\hline $\begin{array}{r}\text { Number } \\
\text { of Samples }\end{array}$ & $\begin{array}{c}\text { Input } \\
\text { power } 5 \mathrm{~mW}\end{array}$ & $\begin{array}{c}\text { Output } \\
\text { power } \mathrm{mW}\end{array}$ \\
\hline 1 & $10 \mathrm{mw}$ & 1.12 \\
\hline 2 & $10 \mathrm{mw}$ & 1.10 \\
\hline 3 & $10 \mathrm{mw}$ & 0.92 \\
\hline 4 & $10 \mathrm{mw}$ & 0.97 \\
\hline 5 & $10 \mathrm{mw}$ & 1.3 \\
\hline 6 & $10 \mathrm{mw}$ & 1.21 \\
\hline 7 & $10 \mathrm{mw}$ & 1.12 \\
\hline 8 & $10 \mathrm{mw}$ & 0.87 \\
\hline 9 & $10 \mathrm{mw}$ & 0.97 \\
\hline 10 & $10 \mathrm{mw}$ & 1.12 \\
\hline 30 & $10 \mathrm{mw}$ & 1.13 \\
\hline
\end{tabular}

Notice:

- With the $650 \mathrm{~nm}$ wavelength $10 \mathrm{~mW}$ laser source passes through, the output power is $1,167 \mathrm{~mW}$. 
- Energy is reduced to $23.34 \%$, the energy output is still ensuring the biological interaction with red blood cells, white blood cells.

- Results after steam sterilization EO. The bag shows to ensure sterile conditions before use.

Figure 7 shows the intravenous optical needle kit.

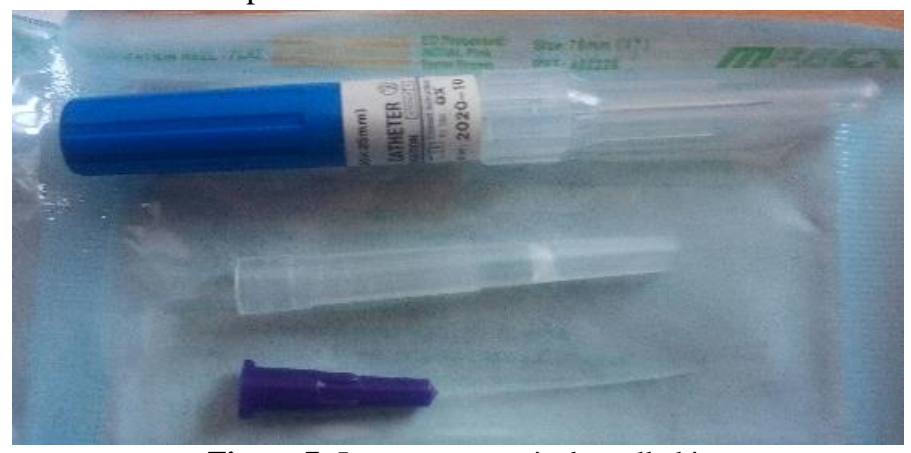

Figure 7: Intravenous optical needle kit

\section{Conclusion}

Intravenous low-level laser therapy is a minimally invasive method in the human epidermis. It has a remarkable effect on the immune system through the $650 \mathrm{~nm}$ wavelength-to-red blood cell and white blood cell:

The optical needle test with low-level laser at $650 \mathrm{~nm}$ wavelength was improved efficiency and reduced the cost of treatment.

All optical needles are used with the catheter and used once per treatment.

Needle and catheter are autoclaved (EO) and packed to avoid infection. Initial results of the trial have been shown to be effective in treating patients with intravascular laser treatment.

\section{References}

(1) Sturla Haslerud, and etc. The Efficacy of Low-Level Laser Therapy for Shoulder Tendinopathy: A Systematic Review and Meta-Analysis of Randomized Controlled Trials. Physiother. Res. Int. (2014) John Wiley \& Sons.

(2) A.P. Gautam et al. Low level laser therapy against radiation induced oral mucositis in elderly head and neck cancer patients-a randomized placebo controlled trial. Journal of Photochemistry and Photobiology B: Biology 144 (2015) p51-56

(3) M.H.Weber, "The Intravenous laser blood irradiation introduction of a new therapy", p641 - p681.

(4) J.H.Theis, Ph.D, D.V.M "Effect of laser Irradiations on Human Erythcytes: considerations Concerning clinical laser Angioplasty"- University of california at Davis, school of medicine, Davis,California, 1983

(5) X.Q. Mi ab, J.Y.Chen ac, Y Cen, Z.J .Liang, L.W .Zhou “A comparative study of 632.8 and 532nm laser irradiation on some rheological factors in human blood in vitro"Jounal of Photochemistry Photobiology",2004 On Topaz MD.FACC.FACP.FSCAI "Laser in Cardiovascular Interventions" Ebook -Springer 